\title{
Cellulite nécrosante descendante infectieuse d'origine dentaire à diffusion mammaire : Analyse de deux cas
}

\section{Descending infection necrotizing cellulitis of dental origin in breast distribution: Analysis of two cases}

Togo S. ${ }^{1}$, Yena $\mathrm{S}^{1}$, Ouattara MA ${ }^{1}$ Saye $\mathrm{J}^{1}$ Sankare $\mathrm{IB}^{1}$, Touré ACS ${ }^{1}$ Maiga IB. ${ }^{1}$ Koné $\mathrm{A}^{1}$, Dakouo DJ ${ }^{1}$ Sanogo Z.Z ${ }^{2}$ Koumaré S. ${ }^{2}$, Koita AK ${ }^{2}$ Camara $\mathrm{M}^{2}$

${ }^{1}$ Service de chirurgie thoracique hôpital du Mali (Bamako, Mali)

${ }^{2}$ Service de chirurgie A hôpital du Point G

Auteur correspondant : Seydou Togo ; Chirurgie thoracique, Hôpital du Mali

E.mail : drseydoutg@ hotmail.com. Tel + 22365511651

\begin{abstract}
Résumé : Les cellulites nécrosantes descendantes d'origine dentaire sont graves et de prise en charge difficile. Leur diffusion se fait classiquement vers le médiastin, les cavités pleurales, voir le cerveau. La diffusion spécifique à la glande mammaire est atypique, rare et peu décrite. Nous rapportons 2 cas colligés en 15 ans de pratique de chirurgie thoracique. Il s'agissait de deux patientes, l'une âgée de 32 ans et l'autre de 25 ans toutes vivaient en milieu rural. Leurs itinéraires diagnostiques et thérapeutiques, les facteurs de risque, les moyens thérapeutiques utilisés et leurs pronostics ont été discutés. Le but de ce travail était de mettre l'accent sur la gravité de cette pathologie et inciter à la mise en œuvre d'une politique de prévention à l'échelle nationale.
\end{abstract}

Mots clés : cellulite, glande mammaire, dentaire.

\begin{abstract}
Cellulitis of dental origin descending necrotizing are serious and difficult management. Their distribution is conventionally done to the mediastinum, the pleural cavities, even to the brain . The specific extension to the mammary gland is atypical, rare and poorly described. We report two cases collected in 15 years of thoracic surgery practice. There were two patients, one aged 32 years and the other 25 years, all lived in rural areas. Diagnostic and therapeutic routes, risk factors, therapeutic means used and their prognoses were discussed. The aim of this work was to emphasize the seriousness of this disease and encourage the implementation of a scale prevention policy.
\end{abstract}

Keywords: cellulitis , mammary gland, dental

\section{1-Introduction}

La cellulite nécrosante descendante d'origine dentaire (CNDD) est une pathologie grave avec une morbi-mortalité élevée pouvant atteindre 18\% [1] Elle est liée à une complication des infections dentaires négligées. La diffusion spécifique à la glande mammaire est exceptionnelle. En 15 ans de pratique de chirurgie thoracique, nous en avons rencontré que 2 cas de cellulite nécrosante descendante cervico-faciale d'origine dentaire. Le but de ce travail est d'expliquer la gravité des CNDD et d'inciter à leur prévention.

\section{2- Observations:}

Cas $\mathbf{n}^{\circ} 1$ : : Il s'agit d'une femme âgée de 32 ans, ménagère, mariée vivant dans une zone rurale d'orpaillage aux antécédents d'avulsion dentaire ( dents 32, 36, 41 et 47) avec port de prothèse dentaire qui a présenté des épisodes de douleurs dentaires chroniques traitées plusieurs fois (médicalement et traditionnellement) en zone rurale. Au mois de mai 2008, elle a présenté une douleur dentaire et des quintes de toux dans un contexte de fièvre associée à une tuméfaction génienne basse. Elle décide sur conseil de sa famille (mère) d'entreprendre un traitement traditionnel fait de massage pour envoutement chez un guérisseur réputé de la place. La tuméfaction génienne basse s'est aggravée secondairement avec une inflammation cervicale qui s'est progressivement étendue aux deux glandes mammaires, successivement le sein gauche puis le droit. Au bout d'une semaine, une perforation des abcès mammaires suivi de nécrose étendue des deux glandes mammaires a été observée (Fig1). C'est devant l'aggravation du tableau couplée à l'installation progressive d'une altération de l'état général que la patiente a été évacuée le 08 juin 2008 au CHU du Point G pour prise en charge. A son admission elle avait une polypnée (30 cycles $/ \mathrm{mn}$ ), une saturation en oxygène (SPO2) à l'air de $95 \%$ et avait un indice de performance OMS à 2 . L'examen physique retrouve une nécrose cervicale étendue au niveau de la glande mammaire avec un délabrement large du tissu mammaire qui est plus accentué au niveau du sein gauche. A l'examen odontologique, on trouve une carie dentaire des molaires inferieures gauches. Le bilan cardiopulmonaire est normal en dehors d'une tachycardie sinusale à 125 battements par minute. Le reste de l'examen clinique était sans particularités 
pathologiques. Une radiographie thoracique réalisée ne retrouve pas d'épanchement intrathoracique. Le bilan biologique retrouve un syndrome infectieux avec une hyperleucocytose à $18000 \mathrm{~mm}^{3} / \mathrm{dl}$, une anémie à $5,3 \mathrm{~g} / \mathrm{dl}$ et une hyperglycémie à $3.4 \mathrm{~g} / \mathrm{dl}$. Sur la base diagnostique d'une CNDD à extension mammaire dans un contexte d'hyperglycémie nous avons procédé au bloc opératoire à une nécrosectomie avec des prélèvements bactériologiques. Le traitement médical a consisté à une triple antibiothérapie probabiliste à base de métronidazole, de gentamycine et de pénicilline $\mathrm{G}$. Ce traitement a été par la suite dirigé par l'antibiogramme qui a isolé le pseudomonas aéruginosa et le staphylocoque aureus, deux germes multirésistants mais sensible à la gentamycine, au betazidime et à l'imipenème. Il a été associé également une extraction dentaire suivie d'un protocole d'hygiène bucco-dentaire et le traitement de l'hyperglycémie. Une greffe de peau a été réalisée 2 mois après que la plaie soit devenue propre. L'évolution a été favorable et l'évaluation de l'état de la patiente à 3 mois, 6 mois puis 1 an après son hospitalisation était satisfaisante.

Cas n'2: Il s'agissait d'une patiente âgée de 25 ans, mariée, ménagère, vivant en zone rurale ayant comme antécédent une carie dentaire à l'enfance, une extraction dentaire des 24,34 et 35 qui remontait à 15 jours chez qui l'infection buccodentaire était la cause d'une cellulite cervico-faciale qui a diffusée spécifiquement dans la glande mammaire gauche . Elle entreprend une automédication avec des traitements médicamenteux non spécifiques, un traitement traditionnel à base de fumigation et de décoction chez un guérisseur de la place. Devant la persistance des symptômes, elle vient en consultation à l'Hôpital du Mali (centre d'appui sanitaire de la coopération médicale chinoise) pour pouvoir bénéficier de traitement médical chinois. Elle est alors orientée au service de chirurgie thoracique pour la prise en charge. A l'admission il existait une tuméfaction cervico-thoracique diffuse, chaude et fluctuante s'étendant sur la glande mammaire gauche avec un syndrome inflammatoire et une tuméfaction génienne basse ( Fig.2). On notait une fièvre à $39.2^{\circ} \mathrm{C}$, une altération de l'état général avec un indice de performance OMS quotté à 2, un pouls à $140 \mathrm{bat} / \mathrm{mn}$, une fréquence respiratoire à 26 cycles/mn, la tension artérielle à $110 / 60 \mathrm{mmhg}$, une $\mathrm{SaPO}_{2}$ à $90 \%$ à l'air ambiant et un taux d'hémoglobine à $8 \mathrm{~g} / \mathrm{dl}$. La Tomodensitométrie (TDM) cervico-thoracique retrouve un abcès des parties molles sous mandibulaire et fascio-cervicale gauche. Cet abcès se prolongeant à la glande mammaire gauche, avec necrose des espaces celluleux et un emphysème sous cutané (Fig. 3). L'examen odontologique trouve une carie dentaire au niveau des molaires inférieures gauches (dents 36 et 37). Un prélèvement est réalisé pour antibiogramme avant d'instaurer un traitement à base de béta-lactamines, d'aminosides et de dérivés imidazolés pendant 3 semaines. L'examen bactériologique du pus est revenu stérile. Au bout de 48 heures de traitement après son admission, on observe une extension de la nécrose cutanée au niveau basi-cervicale, thoracique antérieure et s'étendant à la glande mammaire gauche, avec des poches multi cloisonnées. Une mise à plat des abcès associée à une nécrosectomie est réalisée au bloc opératoire. Une consultation odontologique est réalisée pour l'avulsion des dents causales. Un pansement biquotidien avec nécrosectomie itérative a entrainé une amélioration locale. La cicatrisation définitive est obtenue au bout de 95 jours.

\section{3-Discussion}

L'incidence élevée des cellulites odontogènes est essentiellement due à la mauvaise hygiène buccodentaire. Souvent, un antécédent récent parfois encore évolutif d'avulsion, de soins ou d'abcès dentaires est retrouvé [2] .Cela concorde avec la cause de la cellulite cervico-faciale chez nos 2 patientes. Beaucoup d'auteurs soulignent le rôle favorisant du terrain et en particulier du diabète [3]

En effet, les tares comme le diabète, l'immunodépression (VIH) et certains facteurs liés à la pauvreté comme l'hygiène bucco-dentaire, la malnutrition, l'anémie sont entre autres des facteurs qui grèvent la cellulite [4]. Nos patientes vivaient toutes en milieu rural très paupérisé et aussi dans notre observation, une patiente avait un diabète de type II méconnu et découvert lors des examens biologiques. Ces facteurs auraient favorisé et aggravé la maladie. Chez la patiente diabétique, les lésions de la glande mammaire étaient plus graves, bilatérales et la cicatrisation a été fortement retardée. Ce retard de cicatrisation justifie le recours à la greffe de peau qui nous a permis d'avoir une cicatrisation dirigée. La prise en charge tardive des patientes de notre étude est liée au contexte socio-culturel caractérisé par le recours à la tradithérapie en première intention et aux difficultés financières. Ce constat est celui de plusieurs auteurs qui indiquent la pauvreté dans le milieu africain comme un contexte favorisant le retard de prise en charge sanitaire [5-6] .

Nos patientes n'avaient pas d'obstruction des voies aériennes. Leur dyspnée a été principalement attribuée à l'anémie sévère et à l'hyper catabolisme infectieux. L'hypoxie et la majoration du tableau toxi-infectieux doivent alerter davantage le médecin [2-3]. En effet, toute dyspnée chez un sujet présentant une cellulite cervicale est un signe d'aggravation et doit faire rechercher une extension $\mathrm{du}$ processus infectieux nécrotique vers le médiastin. La radiographie standard et la TDM thoracique ont été réalisées chez nos patientes dans cet objectif mais n'ont pas permis de mettre en évidence des lésions médiastinales ou pleurales. La 
radiographie du thorax permet dans un certains cas de poser le diagnostic de la CNDD par identification d'épaississement des zones cellulitiques et plus précisément en cas de présence de collection. La TDM grâce à son excellente résolution en densité tissulaire et aux renseignements anatomiques qu'elle procure, permet d'apporter des arguments en faveur de la nature inflammatoire d'une tuméfaction cervicale et de rechercher une collection intrathoracique dont la mise en évidence imposerait le drainage chirurgical [6]. A partir de l'infection dentaire, la diffusion de la CNDD peut se faire selon 3 voies de diffusion anatomiques : l'espace pré trachéal (10\% des cas) entrainant les épanchements pleuro péricardiques, l'espace péri vasculaire $(20 \%$ des cas $)$ entrainant une diffusion de poche en poche autour des structures vasculaires et l'espace retro pharyngé ( $70 \%$ des cas) qui est la voie élective entre l'espace cervical et le médiastin postérieur[7]. La voie de diffusion de l'espace périvasculaire de poche en poche serait liée à une diffusion spécifique à la glande mammaire qui dénote du retard de prise en charge médico-chirurgicale. Cette forme est très peu décrite dans la littérature. Lorsque la cellulite évolue sous une forme pseudo phlegmoneuse, elle peut s'étendre par voie péri aponévrotique comme une gangrène. Elle atteint le tissu mammaire, passe très rapidement aux zones déclives en faveur de la pesanteur au tronc jusqu'au pelvis parfois. La gangrène entrainant des épanchements gazeux disséquant les espaces cellulo-aponévrotiques. La flore anaérobie à l'état physiologique est saprophyte de la cavité buccale et de l'oropharynx [8]. A la faveur de modifications locales ou générales, ces germes deviennent pathogènes. $\mathrm{Ce}$ sont donc des germes «opportunistes» [6]. L'infection se développe et s'auto-entretient dans l'espace celluleux entraînant une myonécrose importante qui favorise le métabolisme anaérobie et donc la croissance bactérienne $[8,1]$. Les cellulites cervico-faciales constituent le prototype des infections polymicrobiennes mixtes. Cela concorde avec notre antibiogramme qui a isolé à la fois le pseudomonas aéruginosa et le staphylocoque aureus. La prédominance des germes anaérobies fait l'unanimité des auteurs $[4,8]$.Cette réflexion nous a conduit à conserver le traitement imidazolé pendant la prise en charge. Les protocoles thérapeutiques en matière de prescription de l'antibiothérapie sont variables dans la littérature. Pour la plupart, l'association de référence est une trithérapie : Bétalactamines, Aminosides et Métronidazole [3,6] La trithérapie a été notre protocole d'antibiotherapie probabiliste. En cas de collections suppurées ou de plages de nécrose ; la chirurgie se révèle alors indispensable [7]. Le geste chirurgical doit être aussi complet que possible. Il ne s'agit pas simplement de drainer mais d'exciser la nécrose et mettre à plat toutes les zones cellulitiques. La prise en charge des patients doit être rapide et multidisciplinaire dans le cadre d'une collaboration entre radiologues, bactériologistes, anesthésistes-réanimateurs, nutritionnistes, infectiologues et chirurgiens $[4,8]$. 4-Conclusion

La cellulite cervico-faciale nécrosante d'origine dentaire est une pathologie grave avec une morbimortalité élevée. Elle est fréquente dans les milieux pauvres surtout en Afrique subsaharienne où l'hygiène de vie reste précaire. La diffusion de la cellulite spécifiquement à la glande mammaire est une forme rare. Un traitement basé sur l'antibiogramme et une prise en charge précoce permet d'obtenir de meilleurs résultats. Un accent particulier doit être mis sur la prévention par un programme national de sensibilisation sur les mesures d'hygiène bucco-dentaire dans notre contexte.

\section{Références}

1- Righini CA. Cellulites cervicales extensive et médiastinite descendante nécrosante Ann. Otolaryngol Ch Cervicofac 2007, 124: 292- 300

2- Boca P, Moreau P. Complications cervicales d'infections dentaires : les causes - le diagnostic le traitement. Acta Oto-Rhino-Laryngol 1995, 49 : $37-44$.

3- Kpemissi E. Cellulites cervico-faciales d'origine bucco-dentaire. Etude de 26 cas au CHU de Lomé. Rev Laryngol Oto-Rhinol 1995, 116 : 195-7.

4- Benzarti S., Mardassi A., Ben Mhamed R.et Al. Les cellulites cervico-faciales d'origine dentaire, à propos de 150 cas. Journal Tunisien ORL 2007 Déc , $\mathrm{N}^{\circ} 19$.

5- Razafimanjato NNM., Raoelijaona L., Rasoaamampianina LE. et Al. Médiastinite compliquant une cellulite cervicale d'origine dentaire, à propos d'un cas et revue de la littérature . Rev. anesth réanim med Urgence 2012, 4, (1): 20-22.

6- Boyer JC, Hélénon O, Coste Aet al. Apport de la tomodensitométrie dans l'exploration des suppurations cervicales. Ann Otol-Laryngol Chir Cervico-Fac Paris 1994, 111 : 59-68.

7- Doddoli C., Trousse D, Avaro JP et Al. Traitement des médiastinites nécrosantes descendantes aigues. EMC, Techniques chirurgicales Thorax 2009, $42: 182$

8- Longue Bray A, Legros M, Desphieux JL et al. Cellulites cervico-faciales à germes anaérobies. Les cahiers d'ORL 1983, 18 : 917-22. 


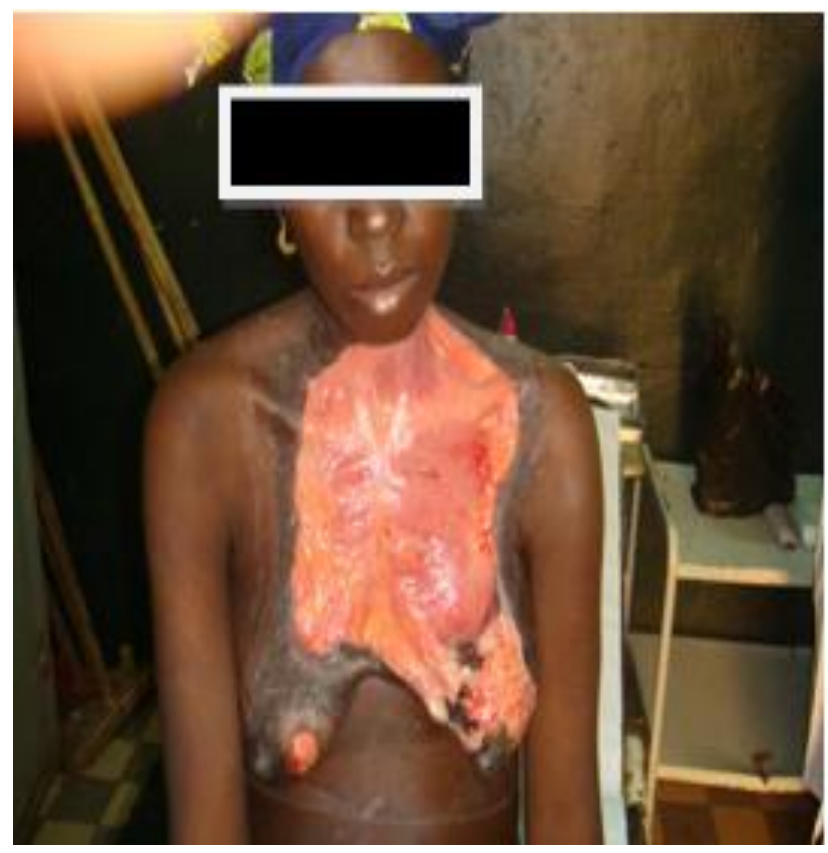

Fig 1: cellulite cervico- faciale avec gangrène et nécrose étendue bilatérale de la glande mammaire

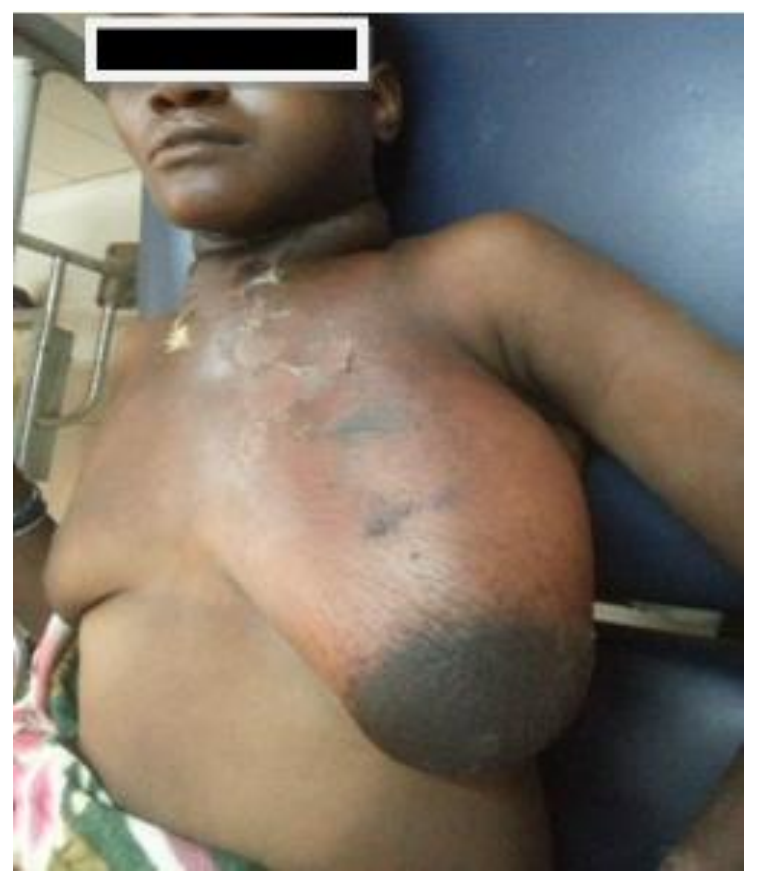

Fig2 : Tuméfaction cervico- faciale et de la glande mammaire gauche 


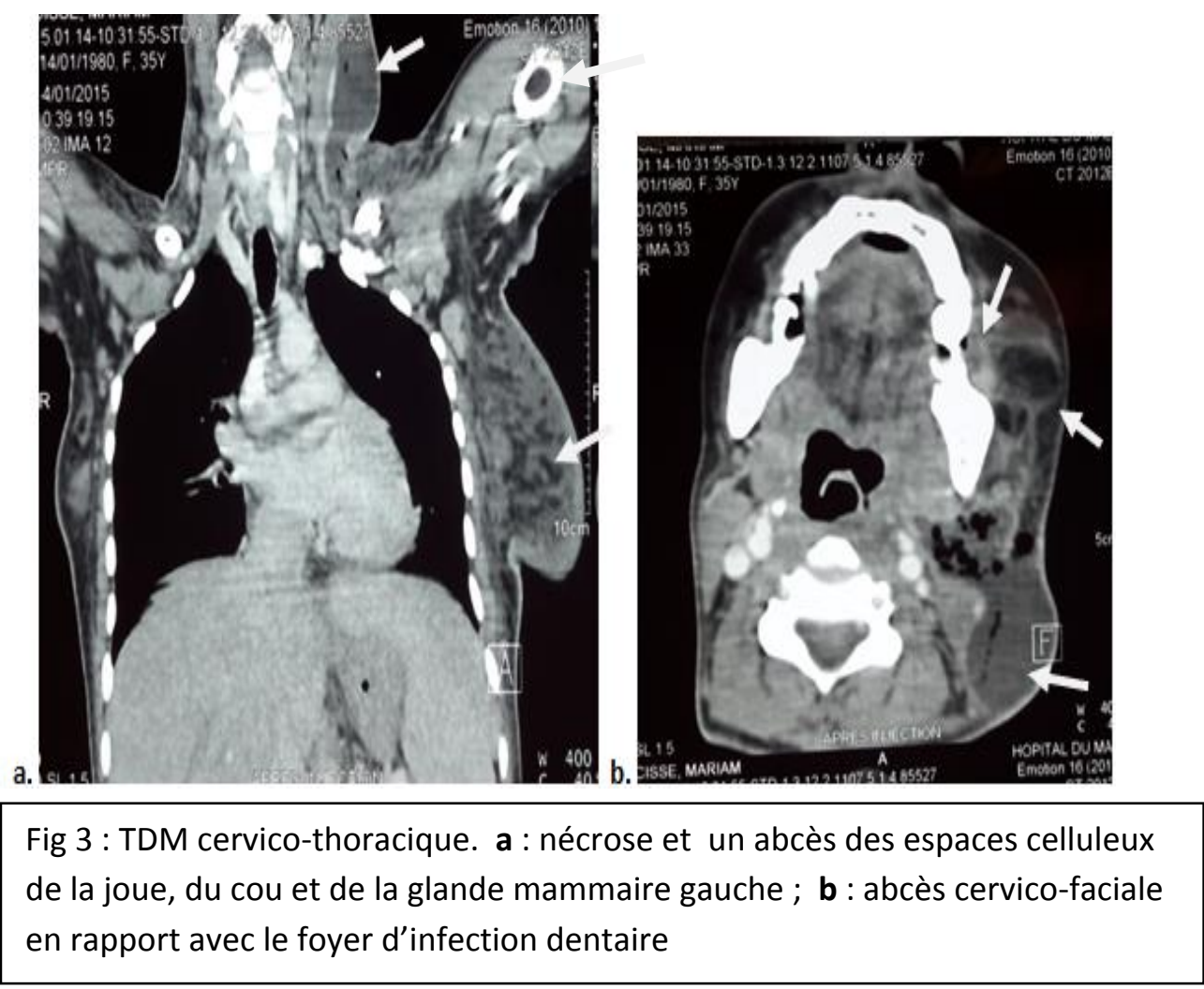

